\title{
IMPROVEMENT OF REJECTION-INDUCED DIASTOLIC ABNORMALITIES IN RAT CARDIAC ALLOGRAFTS WITH INDUCIBLE NITRIC OXIDE SYNTHASE INHIBITION
}

Pablo F. Soto, MD

Chao-Xiang Jia, $\mathrm{MD}^{\mathrm{c}}$

David G. Rabkin, $\mathrm{MD}^{\mathrm{c}}$

Joseph P. Hart, $\mathrm{MD}^{\mathrm{c}}$

Yvonne M. Carter, MD, ${ }^{\mathrm{b}}$

Michael J. Sardo, BS, ${ }^{c}$

Daphne T. Hsu, MD, ${ }^{\mathrm{d}}$

Peter E. Fisher, MD, ${ }^{\mathrm{f}}$

David J. Pinsky, MD

Henry M. Spotnitz, MD
Objective: Inhibition of inducible nitric oxide synthase (nitric oxide II) activity has been proposed as a method to attenuate capillary leak and edema during rejection of heterotopically transplanted rat hearts. Myocardial edema has previously been implicated in diastolic dysfunction during allograft rejection. Accordingly, we tested the hypothesis that inducible nitric oxide synthase inhibition with aminoguanidine would alleviate left ventricular stiffening and myocardial edema formation in 4-day heterotopic rat heart allografts.

Methods: Passive left ventricular filling was studied in American Cancer Institute Lewis rats receiving heterotopic heart transplants receiving either aminoguanidine, a selective nitric oxide synthase inhibitor $(n=6)$; dexamethasone ( $1 \mathrm{mg} \cdot \mathrm{kg}^{-1} \cdot \mathrm{d}^{-1}$ administered subcutaneously) for 4 days after transplantation $(n=6)$; or intravenous saline solution $(n=6)$. American Cancer Institute-to-American Cancer Institute isografts $(n=6)$ were used as controls.

Results: Serum nitrite/nitrate levels in the aminoguanidine group (18 \pm 3 $\mathrm{mmol} / \mathrm{L})$ and dexamethasone group $(22 \pm 4 \mathrm{mmol} / \mathrm{L})$ were reduced versus the intravenous saline group (144 $\pm 36 \mathrm{mmol} / \mathrm{L}$ [SEM]) to levels seen in controls $(25 \pm 9 \mathrm{mmol} / \mathrm{L})$. Left ventricular volume at $15 \mathrm{~mm} \mathrm{Hg}$ for the aminoguanidine group was increased versus that for the intravenous saline solution group, similar to that for controls, and reduced versus dexamethasone-treated animals. Myocardial water content for the aminoguanidinetreated animals $(78.3 \% \pm 0.4 \%)$ was similar to those of intravenous saline-treated animals $(78.0 \% \pm 0.3 \%)$ but greater than those of controls $(77.1 \% \pm 0.2 \%)$ and dexamethasone-treated animals $(76.7 \% \pm 0.3 \%)$.

Conclusions: Nitric oxide II inhibition with aminoguanidine minimizes the reduction in left ventricular filling that is seen with allograft rejection through a mechanism that is not associated with attenuation of myocardial edema. (J Thorac Cardiovasc Surg 2000;120:39-46)
C ardiac transplantation is an accepted mode of therapy for end-stage heart disease; however, allograft rejection continues to be an obstacle to long-term survival. Recent studies have demonstrated the role of nitric oxide in allograft rejection, graft function, and

From the Department of Medicine, University of Michigan, ${ }^{\text {a Ann }}$ Arbor, Mich; Department of Surgery, University of Washington, ${ }^{b}$ Seattle, Wash; and the Departments of Surgery, ${ }^{c}$ Pediatrics, ${ }^{d}$ Medicine, ${ }^{\mathrm{e}}$ and Pathology, ${ }^{\mathrm{f}}$ Columbia University College of Physicians \& Surgeons, New York, NY.

Supported in part by National Institutes of Health grant HL60900, American Heart Association Grant-in-Aid 92153, National Institutes of Health NRSA grant 1 F32 HL09356-1, and a Columbia University Department of Surgery Startup Funds Grant. Dr Pinsky is an Established Investigator of the American Heart Association. related myocardial edema. ${ }^{1-5}$ We have shown previously that myocardial edema is present clinically in cardiac allograft rejection ${ }^{6}$ and that crystalloid coronary perfusion results in edema and predictable commensurate impairment of myocardial filling. ${ }^{7}$ The impaired left

Received for publication Oct 13, 1999; revisions requested Dec 13, 1999; revisions received March 15, 2000; accepted for publication March 15, 2000.

Address for reprints: Henry M. Spotnitz, MD, Department of Surgery, Columbia College of Physicians \& Surgeons, 622 West 168th St, PH 1422, New York, NY 10032

(E-mail: hms2@columbia.edu).

Copyright (C) 2000 by The American Association for Thoracic Surgery

$0022-5223 / 2000 \$ 12.00+0 \quad \mathbf{1 2} / \mathbf{1} / \mathbf{1 0 7 1 2 4}$

doi: $10.1067 / \mathrm{mtc} .2000 .107124$ 
ventricular (LV) filling that is seen in the rejecting cardiac allograft, however, is incompletely explained by myocardial edema. ${ }^{8}$ In the present study, the inducible nitric oxide synthase (iNOS, NOS II) inhibitors aminoguanidine and prednisone were used to further delineate the importance of edema in diastolic abnormalities associated with rejection in rat heart transplantation.

\section{Methods}

Heterotopic rat heart transplantation. All animals received humane care in compliance with the "Principles of Laboratory Animal Care" formulated by the Institute of Laboratory Animal Resources and the "Guide for the Care and Use of Laboratory Animal Resources" prepared by the Institute of Laboratory Animal Resources and published by the National Institutes of Health. Male ACI and Lewis rats were purchased from Harlan Sprague-Dawley (Indianapolis, Ind). Heterotopic abdominal heart transplantation was performed in either ACI-Lewis allografts or ACI-ACI isografts by the technique of Ono and Lindsey ${ }^{9}$ and modified as previously described. ${ }^{8}$ In brief, donor hearts were arrested with cold $\left(4^{\circ} \mathrm{C}\right)$ University of Wisconsin solution and stored in the arrest solution while the recipient animal was prepared. Total ischemic time was kept at less than 30 minutes to minimize the effects of ischemic contracture. After the induction of anesthesia with ketamine $(80 \mathrm{mg} / \mathrm{kg})$ and aseptic preparation of the recipient animal with iodine solution, a midline abdominal incision was made, and the donor aorta and pulmonary artery were anastomosed to the recipient abdominal aorta and inferior vena cava, respectively. The abdominal incision was then closed, and the animals were allowed to recover. Animals were fed a standard diet of water and rat chow ad libitum.

Experimental groups. Four experimental groups were studied: ACI, ACI isografts $(n=6)$, intravenous saline-treated ACI-Lewis allografts $(n=6)$, aminoguanidine-treated allografts $(n=6)$, and dexamethasone-treated allografts $(n=6)$. Immediately after transplantation, intravenous saline-treated ACI-Lewis allograft rats underwent cannulation of the external jugular vein with a plastic catheter that was connected to an ALZA osmotic infusion pump (Alza Corp, Palo Alto, Calif) loaded and primed with $0.9 \%$ normal saline solution and implanted in the subcutaneous space. Aminoguanidinetreated animals were prepared in the same way; however, osmotic pumps were loaded with aminoguanidine hemisulfate (Sigma Chemical Co, St Louis, Mo) dissolved in 0.9\% normal saline solution $(400 \mathrm{mg} / \mathrm{mL})$. Dexamethasone-treated animals received similar osmotic pumps loaded with dexamethasone, which was administered subcutaneously without a catheter at a rate of $1 \mathrm{mg} \cdot \mathrm{kg}^{-1} \cdot \mathrm{d}^{-1}$. Isografts did not undergo pump implantation and were not treated. On post-transplantation day 4 , animals were again anesthetized, and serum was obtained from the external jugular vein for determination of nitrite-nitrate levels. Both transplanted and native hearts were arrested with University of Wisconsin solution, excised, and assessed for LV pressure-volume rejection, myocardial water content, and histology.

Chemiluminescent determination of serum nitritenitrate. External jugular venous blood was centrifuged to remove red blood cells, and aliquots of the remaining serum were reduced to nitric oxide (NO) by using acidic vanadium chloride. Subsequently, the NO was allowed to react with ozone in the reaction chamber of a nitric oxide analyzer (model 280, Seivers). The resultant chemiluminescence is directly proportional to the amount of NO introduced. ${ }^{10}$

Determination of the $L V$ pressure-volume relation. Excised hearts were instrumented with a 16-gauge Angiocath catheter (Becton Dickinson, Franklin Lakes, NJ) stopcock, and $5 \mathrm{~F}$ micromanometer as described previously. ${ }^{8}$ University of Wisconsin solution was infused in $0.02-\mathrm{mL}$ aliquots until an LV pressure of greater than $20 \mathrm{~mm} \mathrm{Hg}$ was reached. The operator performing the volume infusion (C.-X.J.) was blinded to the group assignment of each heart under study. Pressure-volume data were analyzed as described previously. ${ }^{8}$ The mean curve was calculated for each animal by averaging ventricular pressures corresponding to the volume injected.

Myocardial water content. After determinations of LV pressure volume relationship (LVPVR) and removal of the atria, hearts were gently blotted dry, placed on a preweighed piece of foil, and weighed on an analytic balance (model H16; Mettler-Toledo, Hightstown, NJ). Next, hearts were bivalved through both ventricles, and a hemicross section was excised and placed in $4 \%$ formalin for histologic study. The remainder of the specimen was again weighed before and after drying for 48 hours in a $60^{\circ} \mathrm{C}$ oven. Myocardial water content was calculated according to the following equation:

\section{Myocardial water content $(\%)=100 \times(\mathrm{WHW}-$ DHW)/WHW}

where WHW is the wet heart weight and DHW is the heart weight obtained after drying.

Histology. Cardiac cross-sectional samples comprising portions of both left and right ventricles were fixed overnight in $4 \%$ formalin, embedded in paraffin, sectioned, and stained with hematoxylin and eosin. Each slide was graded by a blinded observer for International Society of Heart and Lung Transplantation ${ }^{11}$ rejection grade as follows: rejection grade 0:0, 1A:1.0, 1B:1.5, 2:2.0, 3:3.0, 3B:3.5, 4:4.0.

Statistical analysis. So that LVPVR between animals of different body weights (W) could be compared, LV raw volumes (V) were normalized (Vn) to a body weight of $273.5 \mathrm{~g}$ (mean weight for the series) by the following equation ${ }^{7}$ :

$$
\mathrm{Vn}=\mathrm{V}(273.5 / \mathrm{W})
$$

Pressure-volume data were generated by infusing incremental volumes of fluid into the LV cavity. The data were analyzed by averaging all of the LV volumes that fell within predefined pressure ranges. For the purposes of analysis, the pressure is treated as the independent variable, and the volume is treated as the dependent variable, even though it was 


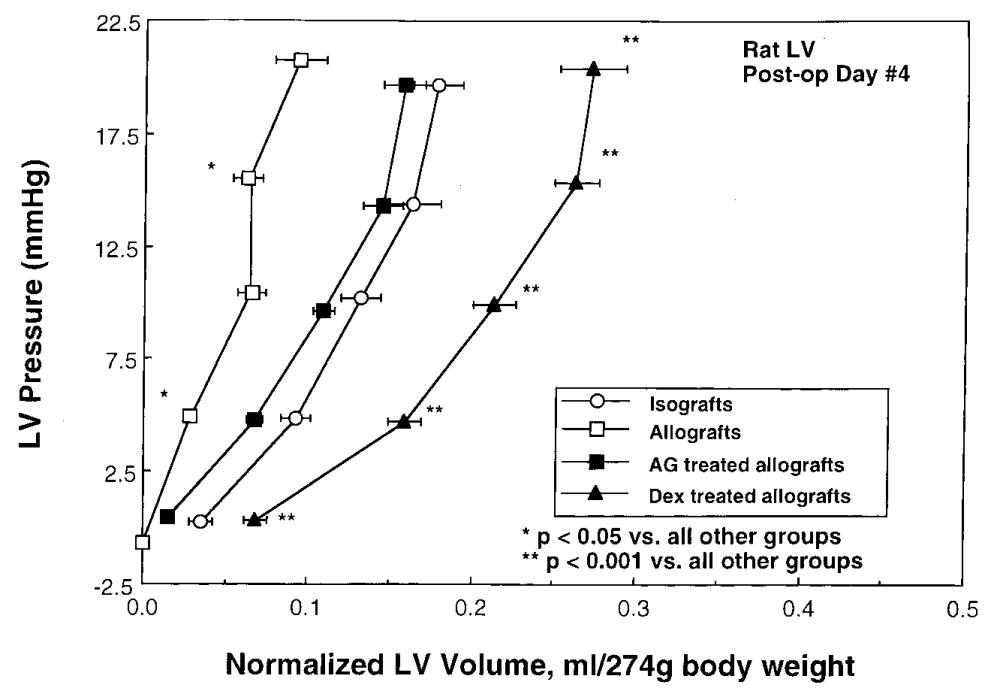

Fig 1. The effect of post-transplantation treatment on diastolic pressure volume curves is demonstrated. At pressure intervals averaging 5 and $15 \mathrm{~mm} \mathrm{Hg}$, allografts had significantly lower filling volumes versus the 3 other groups (aminoguanidine $[A G]$, dexamethasone $[D e x]$, and isografts; $P<.05$ ). Dexamethasone-treated animals had significantly higher filling volumes at all pressure intervals compared with isografts and aminoguanidine-treated animals $(P<.001)$.

the volume that was manipulated experimentally. Pressurevolume data were analyzed by 2 -way repeated-measures analysis of variance (ANOVA), with mean normalized volume within each of 5 pressure ranges averaging $0,5,10,15$, and $20 \mathrm{~mm} \mathrm{Hg}$ as the repeated measure and experimental group as the grouping factor. If significance was found for the group effect or the group-by-pressure interaction, post hoc comparisons of volumes at each pressure range were made among the groups by using the Tukey procedure with a Bonferroni adjustment for the number of comparisons. Mean serum nitrite/nitrate levels and myocardial water content were calculated and compared between experimental groups by 2-way ANOVA and the Tukey posttest.

Mean body weights were compared between groups by 1way ANOVA. Mean percentage myocardial water content were compared by 1-way ANOVA with post hoc comparisons performed by a Bonferroni procedure. Mean edema grades and rejection grades were compared by the KruskalWallis nonparametric ANOVA. Least squares linear regression analysis was performed between individual percentage myocardial water content values and edema grade. Significance in all cases was defined as a $P$ value of less than .05 .

\section{Results}

Reduction in serum nitrite levels in animals treated with dexamethasone and aminoguanidine was measured by serum nitrite to nitrate levels $\left(\mathrm{NO}_{2} / \mathrm{NO}_{3}\right)$. After 4 days of reperfusion, $\mathrm{NO}_{2} / \mathrm{NO}_{3}$ in the intravenous saline-treated ACI-Lewis allograft group
$(144 \pm 36 \mathrm{mmol} / \mathrm{L})$ was significantly elevated when compared with isografts $(25 \pm 9 \mathrm{mmol} / \mathrm{L}, P<.05)$, dexamethasone-treated animals $(22 \pm 4 \mathrm{mmol} / \mathrm{L}, P<$ $.05)$, and aminoguanidine-treated animals (17.5 \pm 2.6 $\mathrm{mmol} / \mathrm{L}, P<.05)$.

The effects of treatment group on the diastolic LVPVR in transplanted and native hearts, respectively, are demonstrated in Figs 1 and 2. For transplanted hearts (Fig 1) at pressure intervals averaging 5 and $15 \mathrm{~mm} \mathrm{Hg}$, the allograft group had significantly lower filling volumes than the other 3 groups (aminoguanidine, dexamethasone, and isografts; $P<.05$ ). Dexamethasonetreated animals had significantly higher filling volumes at all pressure intervals compared with the allograft and aminoguanidine-treated animals $(P<.001)$. Filling volumes in the transplanted hearts were considerably lower than in the native hearts (Fig 2).

The effect of treatment group on myocardial water content is illustrated in Fig 3. Aminoguanidine-treated animals $(78.3 \% \pm 0.4 \%)$ were similar to the allograft group $(78.0 \% \pm 0.03 \%)$ but significantly greater than the isograft group $(77.1 \% \pm 0.2 \%)$ and dexamethasonetreated animals $(76.7 \% \pm 0.3 \%, P<.05)$.

The effect of treatment group on allograft rejection score was assessed. Dexamethasone-treated animals had a significantly lower score $(0.0 \pm 0.0)$ than the allograft group $(3.3 \pm 0.3, P<.001)$ and the aminoguanidine-treated animals $(2.7 \pm 0.4, P<.05)$. 


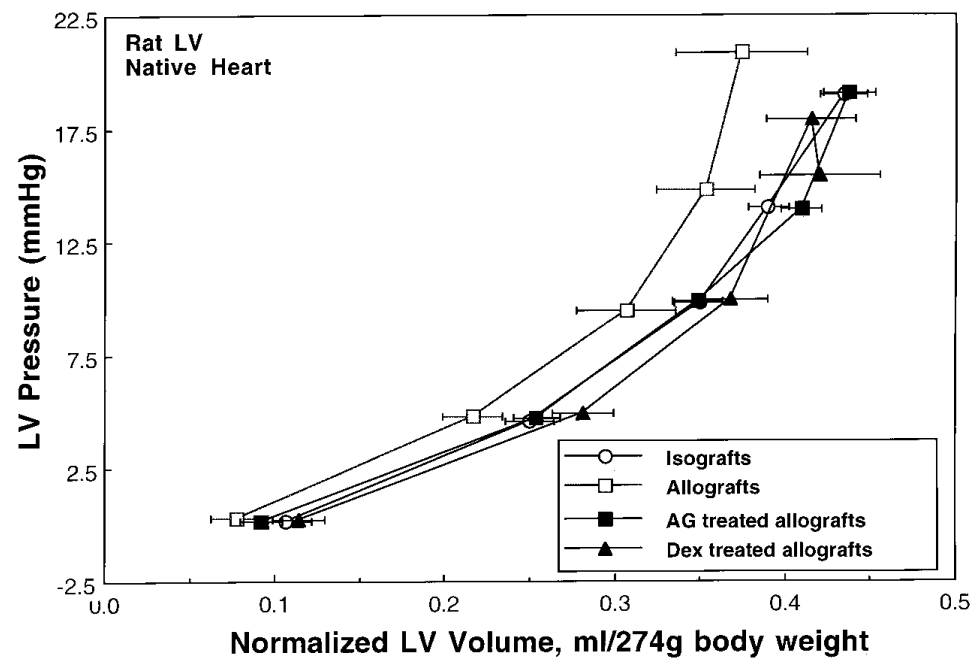

Fig 2. Postmortem LVPVRs for native hearts. No significant differences in normalized LV volume were found among groups. $A G$, Aminoguanidine; Dex, dexamethasone.

Representative hematoxylin and eosin-stained photomicrographs of the experimental groups are displayed in Fig 4. Fig 4, A, shows histologic characteristics of an unmodified isograft displaying relative scarcity of interstitial infiltrate. Fig $4, B$, is an unmodified allograft showing extensive mononuclear cell interstitial infiltration. Fig 4, $C$, is an aminoguanidine-treated allograft showing mononuclear cell interstitial infiltration and increased fiber separation consistent with myocardial edema. Fig 4, D, is a dexamethasone-treated allograft showing no cellular infiltrate and close apposition of fibers.

\section{Discussion}

The constitutive endothelial form of $\mathrm{NO}$ synthase (cNOS, NOS III) is calcium dependent, and NO promotes vasorelaxation. Activity of the inducible form of NO synthase (iNOS, NOS II) is not modulated by ambient calcium levels, is not present under basal conditions, and is induced by rejection and inflammatory mediators. Increased myocardial NOS II messenger RNA, protein, and enzyme activity have been reported in the rejecting heart. Attenuation of this effect of rejection has been achieved previously with immunosuppressive agents, including corticosteroids, cyclosporine (INN: ciclosporin), and FK506. ${ }^{4}$ Worrall and colleagues ${ }^{5}$ demonstrated that specific inhibition of NOS II with aminoguanidine attenuated the histologic changes associated with rejection while minimizing increased capillary permeability and myocardial edema.
The present results augment understanding of the relationship between NOS II and the diastolic properties of transplanted hearts. We demonstrate increased serum $\mathrm{NO}_{2} / \mathrm{NO}_{3}$ levels on day 4 in controls and that steroid immunosuppression on day 4 reduces $\mathrm{NO}_{2} / \mathrm{NO}_{3}$ expression while increasing $\mathrm{LV}$ filling volume and reducing myocardial edema. Aminoguanidine similarly suppresses $\mathrm{NO}_{2} / \mathrm{NO}_{3}$ levels without significantly reducing myocardial edema. LV filling volume, however, increases versus that of controls to a smaller extent with aminoguanidine than with dexamethasone. Aminoguanidine does not reduce lymphocyte infiltration, as measured by the histologic rejection score. Finally, steroids are found to suppress all manifestations of rejection while resulting in diastolic properties superior even to those observed in isografts.

Native heart pressure volume curves were right shifted compared with transplanted hearts, including isografts. Isografts were less compliant than native hearts. Processes in the transplanted hearts that could account for these observations include atrophy because of unloaded state, myocardial edema, or ischemic contracture. Further work is needed to establish the relative importance of each of these processes in this model.

Previous studies of diastolic properties in the arrested heart indicate that ischemic contracture and myocardial edema are the predominant causes of impaired diastolic filling in the arrested left ventricle. However, the present results require an additional mechanism to explain the observed effects of aminoguanidine on fill- 


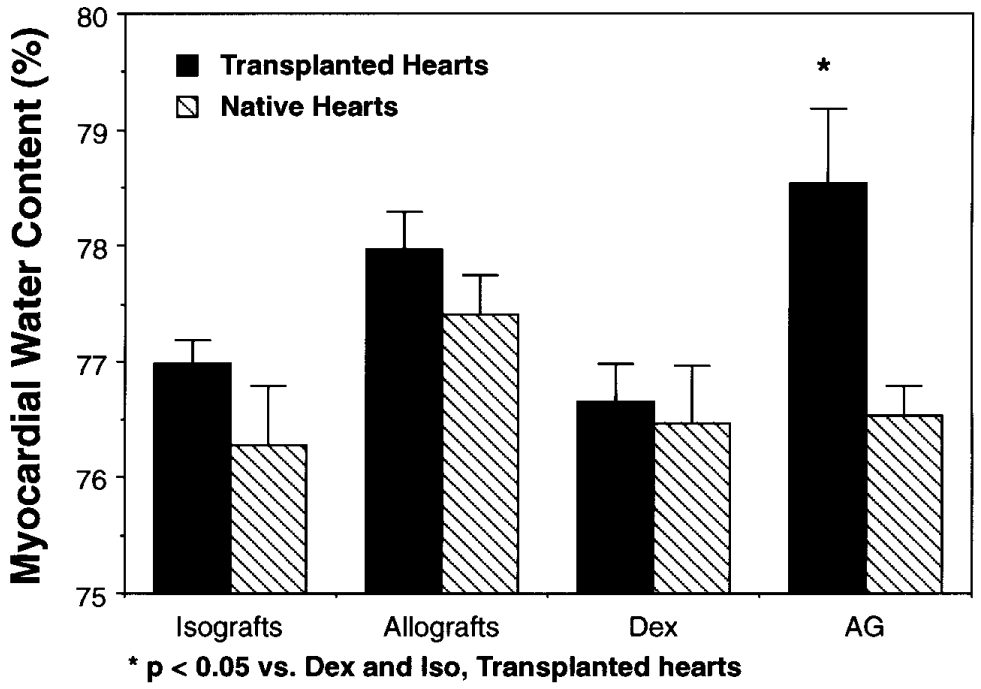

Fig 3. Myocardial water contents for each group. Aminoguanidine $(A G)$-treated animals $(78.3 \% \pm 0.4 \%)$ had similar levels as allografted animals $(78.0 \% \pm 0.03 \%)$ but significantly greater levels than those of isografted animals $(77.1 \% \pm 0.2 \%)$ and dexamethasone $($ Dex $)$-treated animals $(76.7 \% \pm 0.3 \%, P<.05)$.

ing volume. Yuan and colleagues ${ }^{12}$ demonstrated that nitric oxide produced by NOS III augments endothelial monolayer barrier function, preventing retraction of lateral margins of component cells. This prevents paracellular leakage and unrestricted diffusion. Local induction of NOS II in the cardiac allograft in cardiac myocytes and infiltrating macrophages ${ }^{13}$ produces toxic effects and cellular destruction. ${ }^{14}$ Thus NOS II induction and associated augmented NO generation should increase edema in rejecting cardiac allografts.

However, no beneficial effect of aminoguanidine, a relatively selective inhibitor of NOS II, on myocardial water content was observed, although serum $\mathrm{NO}$ and, presumably, NO generation were reduced. Although it is possible that systemic NO was depressed and local NO was not, this is inconsistent with the observation that aminoguanidine reduces the severity of rejection. ${ }^{5}$ One possible explanation for the present findings is that local release of lysosomal enzymes, complement, and toxic oxidants by recruited leukocytes is the primary determinant of endothelial cell damage and edema in rejection. If this is true, NOS II-mediated effects on edema could be overwhelmed by local toxic reactions.

Alternatively, nitric oxide is continually produced in the normal heart, ${ }^{15}$ where it appears to participate in autoregulation of both myocardial inotropy and lusitropy. Recent studies including work by GrocottMason and colleagues ${ }^{16}$ have shown that NO hastens active myocardial relaxation or lusitropy. However, it is important to distinguish lusitropy (active, ATP-dependent, early diastolic relaxation) from passive myocardial stiffness properties as measured by postmortem pressure volume curves in this study, which would exhibit greater correlation with late diastolic function or compliance. Lewis and coworkers ${ }^{17}$ found that increased NOS II messenger RNA expression by means of reverse transcription-polymerase chain reaction correlated with worsened diastolic function by means of Doppler echocardiographic criteria in 16 patients during the first year after cardiac transplantation. ${ }^{17}$ These effects may be mediated by alterations in calcium sensitivity of the contractile elements or alterations in phospholamban-mediated calcium resequestration. In any case, these local modulatory influences appear to be calcium-dependent and related to NOS III activity within the endocardium and microvasculature. ${ }^{14}$ Increased local NOS II production (or its inhibition by aminoguanidine) could result in dysregulation of the homeostatic role of NOS III in normal cardiac lusitropy by either direct or indirect mechanisms. Evidence for interaction between these two isoforms exists in other model systems. For example, Schwartz and coworkers ${ }^{18}$ concluded that $\mathrm{NO}$ autoinhibition mediates NOS II-NOS III interaction in the kidney in a rat sepsis model. ${ }^{18}$

Aminoguanidine, by inhibiting NOS II and reducing local inflammation, could minimize tissue reaction to the graft and fibrosis. This hypothesis is supported by 
A
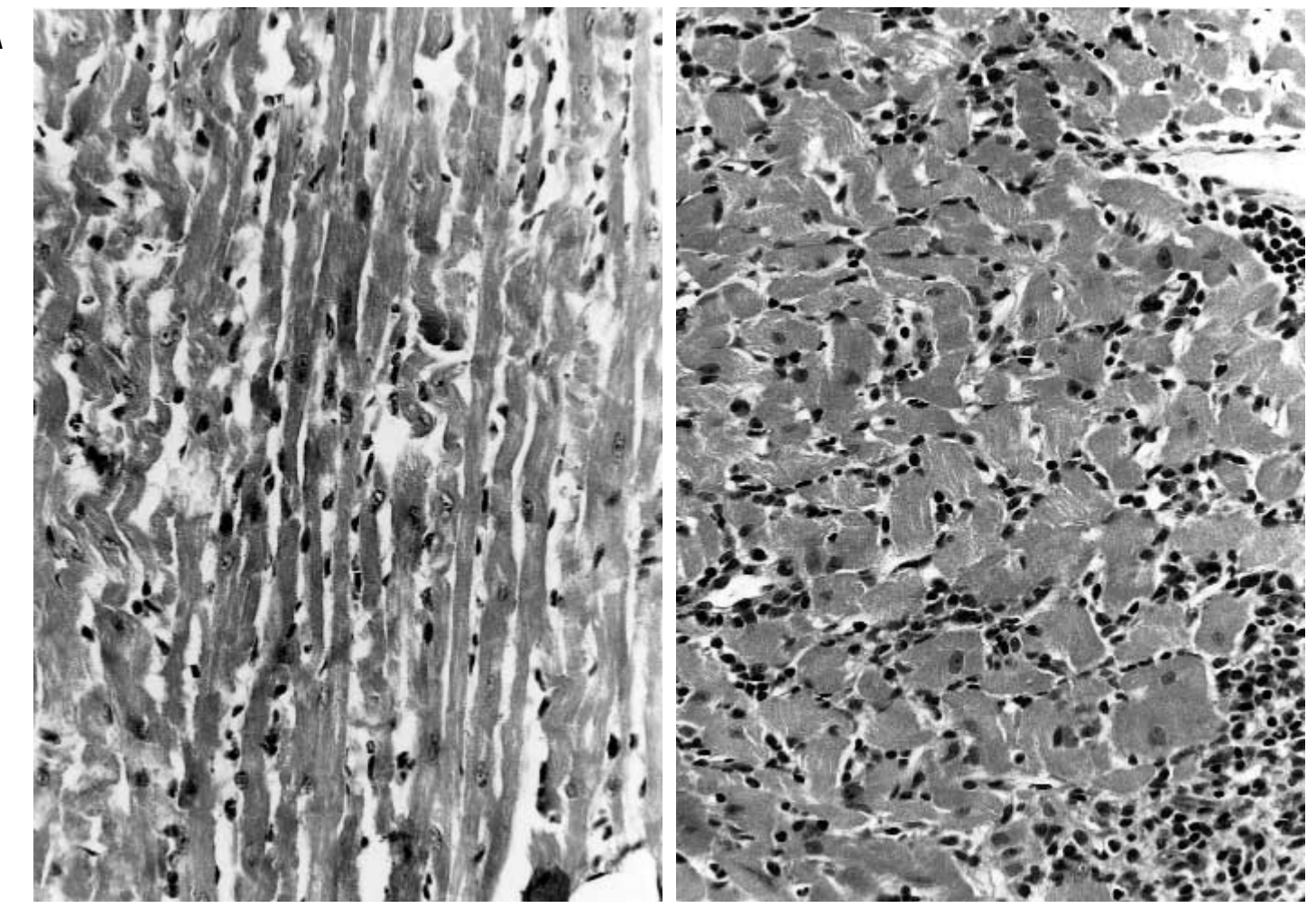

C

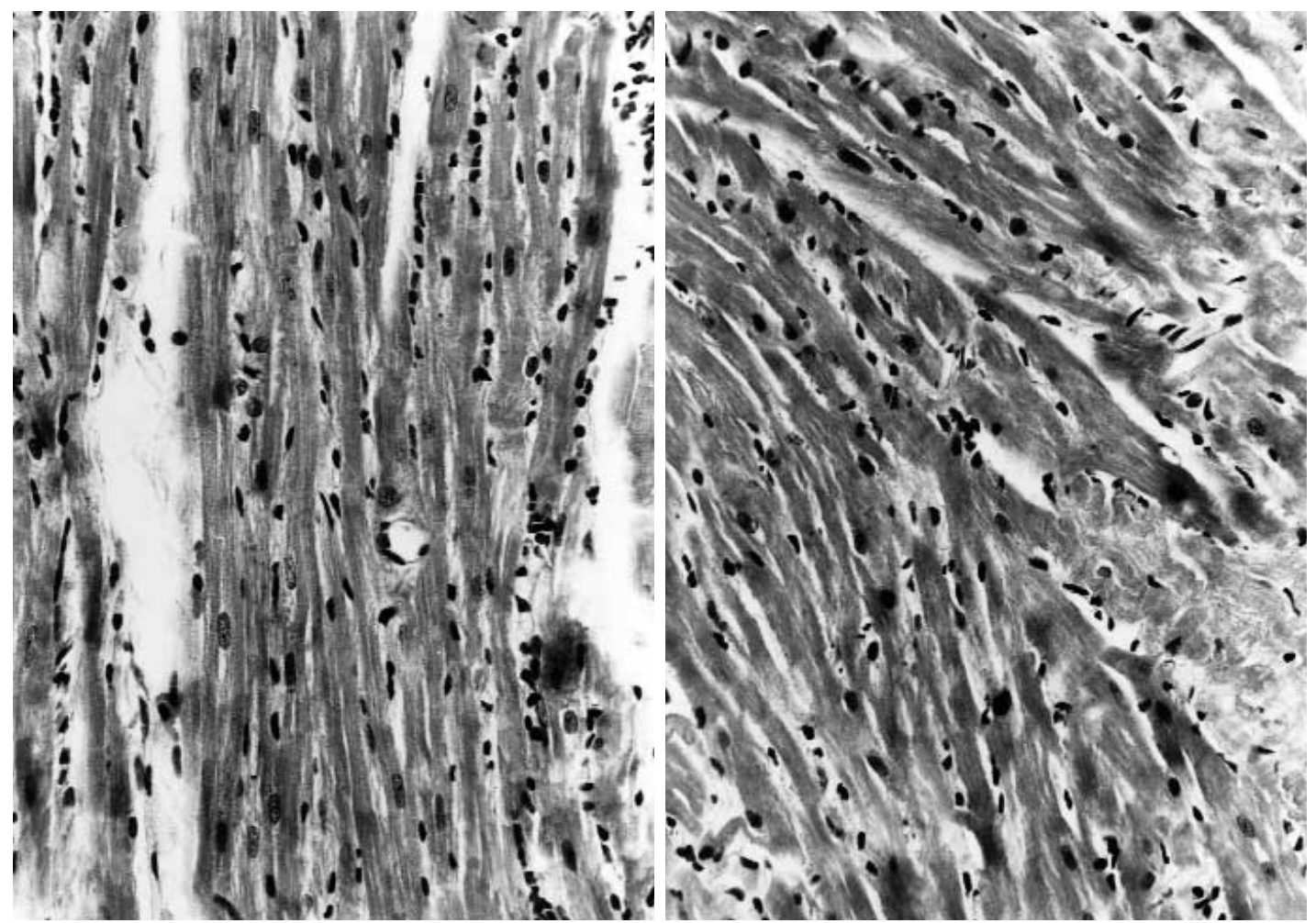

Fig 4. Representative photomicrographs of the experimental groups. A, Isograft myocytes showing relative paucity of interstitial infiltrate. B, Allograft myocytes showing extensive mononuclear cell interstitial infiltration. C, Aminoguanidine-treated myocytes showing interstitial mononuclear cell infiltration and increased fiber separation consistent with myocardial edema. D, Dexamethasone-treated myocytes with no cellular infiltrate and decreased interstitial separation consistent with the absence of myocardial edema. 
the observed effects of steroid administration (which reduced nitrates and water content to isograft levels), normalized rejection score, and eliminated cellular infiltrates. Most important, steroids enhanced filling volume in the allografts to levels superior to those observed even in the isografts. This strongly suggests a generalized effect on fibrosis after ischemic damage at the time of transplantation or on reactive fibrosis at the epicardial surface. With specific respect to compliance in the steroid group, blunting of the inflammatory reaction in the peritoneum after the transplant procedure may have reduced pericardial inflammation and reactive pericardial fibrosis, causing pressure-volume curves in this group to shift further toward those of native hearts. Active fibrosis has not been demonstrated to effect alterations in the pressure-volume relation of the heterotopic isograft, but this could be a factor in this model. Fibrous tissue content was not scored or quantified in the present study, but this may be incorporated into future work on this model. In this scheme we hypothesize two processes adversely affecting diastolic properties of the heterotopic allograft. The first is rejection, which is inhibited to a variable degree by NOS II suppression. The second is fibrosis, which is mediated in the myocardium by inflammation caused by ischemia and reperfusion at the time of surgery and mediated at the epicardial surface by local reaction to surgery. Because all transplanted hearts had reduced filling volumes versus native hearts, it is also possible that myocardial protection during the ischemic period and during reperfusion was inadequate, resulting in diastolic dysfunction. Other performance parameters, such as heart rate, were not assessed in this protocol.

In contradistinction to these data, Worrall and coworkers $^{1}$ found that aminoguanidine inhibition of NOS II in allografts reduced myocardial edema when compared with controls; compliance was not measured by Worrall and coworkers. Differences between the data from this study and those from work by Worrall could be accounted for by numerous experimental factors. That group used wet/dry weight ratios to assess myocardial water content, whereas we used the percentage of myocardial water content. Worrall's group used topical iced saline solution to effect cardiac arrest, whereas we used intracoronary University of Wisconsin solution to induce arrest, and myocardial protection strategy can alter the percentage of myocardial water content. ${ }^{19}$ Differences in tissue processing, including blotting, trimming of RV and atrial tissues, and drying protocols, might also alter results. Correlation of our study with Worrall's data emphasizes that two pathways of aminoguanidine NOS II inhibition of diastolic dysfunction may exist in the heterotopic cardiac transplant model, one dependent on myocardial edema and one independent of myocardial edema. Indeed, recent data indicate that NO triggers programmed cell death (ie, apoptosis) of adult rat ventricular myocytes. ${ }^{20}$ Apoptosis triggers cell death by means of a noninflammatory mechanism; its suppression (through NOS II inhibition) might theoretically preserve compliance. The duration of the current observation period (4 days) is not sufficient to make longterm compliance assessment, which may change with fibrosis and delayed cellular infiltration. However, by 4 days, NOS II blockade does preserve compliance in the present study.

In summary, suppression of NOS II with aminoguanidine restores diastolic properties of rejecting allografts to levels comparable with those of isografts but does not eliminate myocardial edema or histologic manifestations of rejection. Steroid suppression normalizes all manifestations of rejection except for diastolic properties, which are rendered superior to those of isografts, approaching levels appreciated in native ventricles. These observations are consistent with the view that abnormalities of diastolic properties in heterotopic allografts and isografts involve rejection, as well as fibrosis related to ischemia and reperfusion at surgery and reaction at the interface of the epicardium and surrounding tissues. Inhibition of NOS II appears to favorably affect both sources of abnormality of diastolic properties.

\section{REFERENCES}

1. Worrall NK, Chang K, Suau GM, Allison WS, Misko TP, Sullivan PM, et al. Inhibition of inducible nitric oxide synthase prevents myocardial and systemic vascular barrier dysfunction during early cardiac allograft rejection. Circ Res 1996;78:769-79.

2. Worrall NK, Pyo RT, Botney MD, Misko TP, Sullivan PM, Alexander DG, et al. Inflammatory cell-derived NO modulates cardiac allograft contractile and electrophysiological function. Am J Physiol 1997;273(Suppl):H28-37.

3. Worrall NK, Misko TP, Botney MD, Sullivan PM, Hui JJ, Suau GM, et al. Time course and cellular localization of inducible nitric oxide synthases expression during cardiac allograft rejection. Ann Thorac Surg 1999;67:716-22.

4. Cai B, Roy DK, Sciacca R, Michler RE, Cannon PJ. Effects of immunosuppressive therapy on expression of inducible nitric oxide synthase (NOS II) during cardiac allograft rejection. Int J Cardiol 1995;50:243-51.

5. Worrall NK, Lazenby WD, Misko TP, Lin TS, Rodi RP, Manning PT, et al. Modulation of in vivo alloreactivity by inhibition of inducible nitric oxide synthase. J Exp Med 1995;181:63-70.

6. Amirhamzeh MMR, Jia C-X, Starr JP, Sciacca R, Chowdhury $\mathrm{NC}$, Hsu DT, et al. Diastolic function in the heterotopic rat heart transplant model: effects of edema, ischemia, and rejection. J Thorac Cardiovasc Surg 1994;108:928-37.

7. Carter YM, Jia C-X, Soto PF, Starr JP, Rabkin DG, Hsu DT, et al. Diastolic properties, myocardial water content and histologic condition of the rat left ventricle: effect of varied osmolarity of a coronary perfusate. J Heart Lung Transplant 1998;17:140-9.

8. Soto PF, Jia C-X, Carter YM, Rabkin D, Starr JP, Amirhamzeh 
$\mathrm{MM}$, et al. Effect of improved myocardial protection on edema and diastolic properties of the rat left ventricle during acute allograft rejection. J Heart Lung Transplant 1998;17:608-16.

9. Ono K, Lindsey ES. Improved technique of heart transplantation in rats. J Thorac Cardiovasc Surg 1969;7:225-9.

10. Zeballos GA, Bernstein RD, Thompson CI, Forfia PR, Seyedi N, Shen W, et al. Pharmacodynamics of plasma nitrate/nitrite as an indication of nitric oxide formation in conscious dogs. Circulation 1995;91:2982-8.

11. Billingham ME, Cary NR, Hammond ME, Kemnitz J, Marboe C, McCallister HA, et al. A working formulation for standardization of nomenclature in the diagnosis of heart and lung rejection: heart rejection study group. J Heart Transplant 1990;9:587-93.

12. Yuan Y, Granger HJ, Zawieja DC, DeFily DV, Chilian WM. Histamine increases venular permeability via a phospholipase CNO synthase-guanylate cyclase cascade. Am J Physiol 1993;264(Suppl):H1734-9.

13. Yang X, Chowdhury N, Cai B, Brett J, Marboe C. Induction of myocardial nitric oxide synthase by cardiac allograft rejection. $\mathrm{J}$ Clin Invest 1994;94:714-21.

14. Pinsky DJ, Cai B, Yang X, Rodriguez C, Sciacca RR, Cannon DJ. The lethal effects of cytokine-induced nitric oxide on cardiac myocytes are blocked by nitric oxide synthase antagonism of transforming factor beta. J Clin Invest 1995;95:671-85.
15. Pinsky DJ, Patton S, Mesaros S, Brovkovych V, Kubaszewski E, Grunfeld S, et al. Mechanical transduction of nitric oxide synthesis in the beating heart. Circ Res 1997;81:372-9.

16. Grocott-Mason R, Anning P, Evans H, Lewis MJ, Shah AM. Modulation of left ventricular relaxation in isolated ejecting heart by endogenous nitric oxide. Am J Physiol 1994;267 (Suppl):H1804-13.

17. Lewis NP, Tsao PS, Rickenbache PR, Xue C, Johns RA, Haywood GA, et al. Induction of nitric oxide synthase in the human cardiac allograft is associated with contractile dysfunction of the left ventricle. Circulation 1996;93:720-9.

18. Schwartz D, Mendonca M, Schwartz I, Xia Y, Satriano J, Wilson $\mathrm{CB}$, et al. Inhibition of constitutive nitric oxide synthase (NOS) by nitric oxide generated by inducible NOS after lipopolysaccharide administration provokes renal dysfunction in rats. J Clin Invest 1997;100:439-48.

19. Starr JP, Jia CX, Rabkin DG, Amirhamzeh MM, Hart JP, Hsu DT, et al. Pressure volume curves in arrested heterotopic rat heart isografts: role of improved myocardial protection. J Surg Res 1999;86:123-9.

20. Pinsky DJ, Aji W, Szabolcs M, Athan ES, Liu Y, Yang YM, et al. Nitric oxide triggers programmed cell death (apoptosis) of adult rat ventricular myocytes in culture. Am J Physiol 1999;277 (Suppl):H1189-99. 